\title{
History of EPR Studies from the H. M. Swartz Laboratories: Part 3-EPR Oximetry
}

\author{
Harold M. Swartz ${ }^{1}$ (D)
}

Published online: 11 January 2022

(C) The Author(s), under exclusive licence to Springer-Verlag GmbH Austria, part of Springer Nature 2021

\section{Introduction}

I have pursued three distinctly different but sometimes overlapping areas of research on oxygen using EPR as a primary tool, currently totaling about 250 papers and reviews:

- The effects of oxygen on physiological and pathophysiological processes including the deleterious effects of inappropriate levels of oxygen;

- The development and application of methods to measure oxygen in viable biological systems, with an emphasis on potential clinical applications; and

- Instrumental/technique developments to facilitate EPR oximetry, including for clinical use.

\section{Review of research on oxygen from HMS' Laboratories}

This historical review of research on oxygen from my laboratories considers each area separately, while recognizing the frequent overlap that occurs in individual studies.

\subsection{Effects of oxygen on physiological and pathophysiological processes}

\subsubsection{The role for EPR oximetry and early research into viable systems}

The rationale for measuring oxygen is based on the important role that oxygen has in both physiology and pathophysiology. The niche for EPR oximetry comes from the ability of EPR to make measurements of oxygen directly in cells and tissues and, with the use of particulate paramagnetic sensors whose spectra reflect the amount of

Harold M. Swartz

Harold.Swartz@Dartmouth.edu

1 Department of Radiology, Geisel School of Medicine, Dartmouth College, Hanover, NH, USA 
oxygen, the measurements can be made repeatedly. These characteristics underlying the following descriptions of our measurements of oxygen.

My interest in oxygen began early in my research career at the Walter Reed Army Institute for Research, initially as part of the main research that I was asked to carry out on trying to elucidate the mechanism of the radioprotective drugs being developed in a very large radiation protector project ${ }^{5,8,9,10}$. (This project had the goal of developing drugs that could be given to military personnel before exposure to radiation from warfare, so that their capabilities would not be unacceptably diminished).

\subsubsection{The 'oxygen effect' to increase damage from ionizing radiation in biological systems}

My initial research was to try to use a system developed previously by Smaller and Avery [1] to apply EPR to examine energy transfer from irradiated yeast to potential radiation-protection drugs, which had $\mathrm{SH}$ groups as their active component. My aim was to repeat their experiments and expand upon them by relating the observed changes in free radicals to survival of the irradiated yeast. To accomplish this, I used systematic variants of the drugs with known different radioprotective efficacy administered to irradiated mice. I planned to do the experiments in the presence and absence of oxygen because of the well-known 'oxygen effect', i.e., low levels of or no oxygen present in tissues appears to protect cells from damage due to ionizing radiation; (the oxygen effect is discussed in more detail below).

The experimental system was complicated because, to stabilize the radiationinduced free radicals which were expected to be very short-lived in a liquid environment, the experiment needed to be carried out with deeply frozen organisms, i.e., irradiation and initial EPR studies were done at $77 \mathrm{~K}$. At this temperature, the hydroxyl free radicals produced in water, which ordinarily could contribute to biological damage by diffusing to the sensitive sites (termed the indirect effect), cannot cause damage because they are immobilized. Thus, the initial free radicals that are seen in frozen samples of the organisms would be due only to the direct effect on the target molecules.

Because the purpose of the radioprotective drugs was to reduce damage and increase survival following exposure to radiation, I wanted to determine if there was a correlation between scavenging of the radicals and better survival. However, when I measured viability in the yeast that had been irradiated while frozen and then thawed to measure survival, I found that less than $1 \%$ survived the freezing process even without irradiation. I therefore added a second system, the bacterium, E. coli, which had high survival after freeze-thawing $(85 \%)$.

I then carried out parallel experiments with both types of organisms, to probe the impact of the radioprotective drugs on their survival following exposure to freezing and how the outcomes related to the amount and type of radiation-induced free radicals. Because of the importance of oxygen in radiation damage (the presence of oxygen sensitizes cells to the effects of radiation), both systems were studied in the presence and absence of oxygen. The experiments with E. coli provided the most useful data because the impact on free radical generation could be related to changes 
in survival. Two types of control experiments were made on the E. coli: frozen samples, not irradiated and unfrozen samples that were irradiated.

\subsubsection{Principal Findings Related to Oxygen in the Early Studies}

The following summarizes the oxygen-related results of these early studies; (see Part 1 of my laboratories' research ${ }^{573}$ for the other results). First, we found that the presence of oxygen during irradiation impacted the amount and type of radiationinduced free radicals that were observable and, as expected, had a deleterious effect on survival after irradiation. At least some of the oxygen effect occurred via modifying the direct effect, because (as noted above) at $77 \mathrm{~K}$ the hydroxy radicals were immobilized, i.e., the indirect effect was suppressed.

The fact that an effect from the presence of oxygen was observed also confirmed that oxygen was in the immediate vicinity of the radiosensitive target, because molecular oxygen also is immobilized at $77 \mathrm{~K}$ and would not have moved into the sensitive site. We also showed that the presence of the prototypic radiation protective drug beta-mercaptoethylamine (MEA) reduced the amount of oxygen-dependent radiation-induced free radicals in the organism in the presence of oxygen, i.e., it modified the direct effect in regard to the observable yield of free radicals. The data on survival of irradiated samples indicated that the presence of MEA in the frozen suspension reduced the amount of cell killing in the presence (and absence) of oxygen.

What was unexpected was what happened in the unirradiated controls. We found that freezing damage in terms of survival of cells was greater when oxygen was present, but that damage was lessened in the presence of MEA. Although the unexpected results were preliminary, they suggested that there might be another type of 'oxygen effect' on biological systems related to cell damage due to freezing. With the stimulus of this preliminary indication that the presence of oxygen also affected freezing damage, I surmised that there might be a unifying basis for many toxic effects of oxygen. But I recognized that this area of inquiry would make an excellent $\mathrm{Ph} . \mathrm{D}$. thesis topic and therefore chose not to pursue it immediately.

\subsubsection{A Unifying Theme to Understand The Toxicity of Oxygen in the Presence of Injury}

Soon after, I arranged to start my Ph.D. After looking at several alternative programs to pursue my Ph.D., I began in the program at Georgetown U. in Biochemistry/Biophysics, which enthusiastically agreed with the importance of my proposed topic. My Ph.D. thesis was centered on rigorously characterizing this previously unknown type of toxic oxygen effect, namely on freezing damage ${ }^{7}$. The subsequent series of papers comprised my thesis and showed that: the presence of oxygen increased damage from freezing of $E$. coli ${ }^{11}$; the oxygen effect in freezing resulted in modification of the radiation induced free radicals, damage to DNA, and damage to membranes ${ }^{14}$; and MEA could ameliorate the oxygen-dependent part of freezing damage ${ }^{15}$.

Pursuing a doctorate also gave me an opportunity to explore more deeply why oxygen had toxic effects after many types of injury, and I was able to develop a 
satisfying generalized theory ${ }^{26}$. During the course of looking more deeply into the literature, I discovered I was not the first person to try to find a unifying theory for the toxicity of oxygen, so my "discovery" of oxygen's toxicity was not quite as momentous as I had thought. But I believe I added significantly to the overall concept, and in the process learned much more about the role of oxygen in pathophysiology.

The idea behind the integrated theory is simple and logical. Briefly, life on earth is based on maintaining the integrity of very oxidizable biomolecules which need to co-exist with an atmosphere that has high amounts of oxygen, a very potent oxidizer. Energy production in cells utilizes the reaction of carbohydrates with oxygen, but this process needs to be highly controlled so that excess oxidations do not occur. In sum, there is a delicate equilibrium between dealing with oxygen to fuel cellular activity and preventing undesirable oxidations from occurring (the latter is maintained by physical barriers and anti-oxidative processes). It is not surprising that, when injuries occur to compromise this equilibrium, toxicity results. The basis of this hypothesis is contained in an early paper of $\operatorname{mine}^{26}$ and I believe is still correct, although in the intervening years more details have emerged.

\subsubsection{Using Spin-Traps to Study the Toxicity Of Oxygen}

The next set of experiments on deleterious effects of oxygen used the emerging technique of spin-trapping. (Note: the topic of spin trapping per se is considered in detail in the companion paper on the history of research on free radicals ${ }^{573}$.) We demonstrated that, in the presence of aerobic metabolism of nitro-compounds, the potentially toxic intermediate superoxide was produced ${ }^{51}$.

\subsection{The development and application of methods to measure oxygen in viable biological systems, with an emphasis on potential clinical applications}

\subsubsection{Using nitroxide metabolism to enhance information on oxygen from measurements with EPR and NMR techniques}

Contrast agents for NMR are usually paramagnetic molecules. Therefore, the structural versatility of nitroxides has led to their use as NMR contrast agents, but their use was limited because their metabolism to non-paramagnetic products via redox reactions led to the loss of contrast in vivo. I recognized that the oxygen dependency of the metabolism of nitroxides could be exploited as a type of functional NMR in which the loss of contrast reflected metabolism. This also could be a potentially powerful tool to measure oxygen with EPR.

We therefore carried out an extensive set of studies to determine the relationship between structures of nitroxides and their distribution and metabolism and the physical properties of oxygen. These results were then applied to make measurements of function with NMR and EPR 92-95, 97, 99, 108,115, 116, 118, 120-125, 128, 132, 135, 
137, 140, 143-145, 148, 149, 152, 154-156, 158, 160-162, 164, 169, 187, 191, 196, 198, 199, 209, 226 [book], 235, $252,254-263,267,279,302,307,309,364,367,379,403,467$. We also extended this to other types of contrast agents including oxygen itself, dextran magnetite, and nitroxides bound to albumin $^{82,136,147 .}$

\subsubsection{Studies Combining NMR Techniques and EPR Oximetry}

I had the good fortune that Paul Lauterbur felt EPR had high potential for in vivo studies. So, when he formed the leading NMR Society, he said that instead of having NMR in the name, it should be called the Society for Magnetic Resonance in Medicine (SMRM) to include EPR. He included me as the (sole) representative of EPR on the first Board of Trustees, and I stayed on for 10 years in the capacity of Secretary of SMRM. As a consequence, for more than a decade I had the opportunity to work closely with many leaders in NMR, including three who won Nobel Prizes for their work with NMR (Paul Lauterbur, Peter Mansfield, and Kurt Wüthrich). These connections, combined with having a cutting-edge in vivo NMR instrument for making measurements in animals co-located with our EPR laboratory at Dartmouth, led to a considerable number of productive studies in collaboration with outstanding NMR scientists.

Most of our studies combining EPR and NMR focused on oxygen, because both techniques provide uniquely useful and complementary in vivo information on oxygen-related physiology and pathophysiology ${ }^{320}$. We used such a combined approach for what I still believe is one of our most important contributions: the concept of measuring function with NMR by exploiting the oxygen dependent metabolism of nitroxides through the use of nitroxides as contrast agents. In particular we showed, in both model systems and cellular systems, that by using oxygen dependent metabolism of nitroxides and also in some cases the solubility of nitroxides and oxygen, NMR images could detect hypoxia in general and also in specific compartments (e.g., in lipid rich areas) ${ }^{82,87,88,93,94,95}$.

We also carried out several studies in which we used the power of EPR oximetry to enhance the value of NMR techniques aimed at probing oxygen-related physiology and pathophysiology. In particular we started on a theme that continues today: exploiting the specificity of EPR measurements of oxygen to determine the conditions under which more widely available techniques, especially NMR, might provide useful insights on oxygen-dependent phenomena even though the NMR techniques do not directly measure oxygen in tissues ${ }^{541}$.

Using such an approach we showed the relationship between EPR oximetry and NMR diffusion-weighted imaging (DWI) in tumors and were able to indicate the conditions under which DWI might be most useful for this purpose ${ }^{236,266}$. Using 31P-NMR spectroscopy and EPR oximetry we were able to determine the relationship between oxidative metabolism in the brain and local oxygen levels ${ }^{313}$. In collaboration with Towner, we used a combination of MRI, MR spectroscopy (MRS), and EPR oximetry to determine the mechanism of action of Nodularin, a cyclic hepatotoxin isolated from the cyanobacterium Nodularia spumigena ${ }^{351}$. This is an important toxin to humans that can occur at beaches and is especially a problem in Australia. 


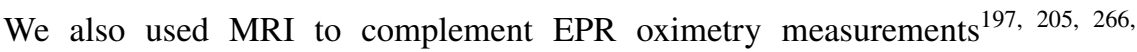
341 . We recognized that NMR techniques could be very useful for expanding on the value of measurements of oxygen with EPR, e.g., by providing data about the location of EPR sensing paramagnetic materials. Using MRI pulse sequences that were impacted by the effects of paramagnetic materials on susceptibility, we could accurately locate the position of the oxygen sensing material in a tumor ${ }^{197,246}$. We also used the different parameters related to oxygen that are measured by NMR to increase understanding of the significance of oxygen measurements by EPR. For example, we measured perfusion and vessel integrity with NMR Gd-DTPA contrast in tumors on which we used EPR with fusinite to measure oxygen ${ }^{205}$.

We especially carried out parallel NMR and EPR studies of oxygen dependent phenomena, adding to the utility of both techniques. In an investigation of oxygen levels in the medulla and cortex of the kidney during septic shock, using BOLD NMR we were able to show that the increase in oxygen measured by EPR was due to increased delivery mediated by nitric oxide which caused vasodilation. We also used BOLD in combination with EPR oximetry to measure the impact on tissue oxygen from breathing carbogen ${ }^{341}$. We showed the relationship between tissue oxygen measured by EPR and NMR diffusion-weighted imaging ${ }^{266}$ and BOLD $^{229,} 282$.

\subsubsection{EPR Imaging of Oxygen and Measurements of Diffusion of Oxygen}

Inspired by the studies related to NMR imaging etc. and oxygen, we also looked at the feasibility of using EPR methodology to directly image oxygen and/or measure its diffusion. We were motivated by the presence in the lab of colleagues from (former) Yugoslavia with appropriate expertise in imaging, especially Franci Demsar and Goran Bačić ${ }^{100,113,117}$. Later, we were able to collaborate with Paul Lauterbur ${ }^{126}$ who had moved to the U. of Illinois at Urbana, in part motivated by having his laboratory collocated with the EPR laboratory.

We developed spatially resolved EPR diffusion and imaging using nitroxides. We measured oxygen by its effects on the spectra and the metabolism of nitroxides. The latter enabled us to combine measures of redox metabolism with measures of oxy-

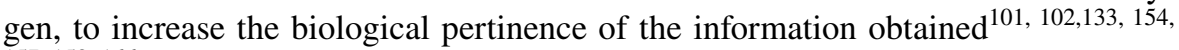
$157,158,166$

We also developed an alternative to full imaging which we termed "multisite spectroscopy", which enabled us to simultaneously obtain EPR spectra from multiple sites using magnetic field gradients ${ }^{189}$, 207, 354, 381, 407, 433, 438, 464, 471, 472, 499, 500, 502 . Alex Smirnov and Oleg Grinberg led these technical developments; later Ben Williams contributed important additions.

The initial studies used separate independent implants of lithium phthalocyanine (LiPc) crystals. Later these concepts were incorporated into a vital development, called the "implantable resonator" (ImR). The ImR has multiple sensing sites held in a fixed array as well as other advantages detailed below. Nadeem Khan and Huagang Hou led the biological applications of these techniques, especially the ImR, which has become the cornerstone of ongoing clinical applications of EPR for oximetry for improving therapy of cancer. 


\subsubsection{Measurements of Oxygen to Study Physiology and Pathophysiology in Isolated Cells}

Initially our studies in living systems were done at X-Band, which has the problem of non-resonant absorption of microwaves, i.e., causing heating of lossy tissues. We were therefore limited to measuring isolated cells in sample holders that could overcome this problem, i.e., by making measurements in EPR cavities in the regions where there was minimal electrical field and maximum magnetic field. We used flat cells oriented in the plane of minimal electrical intensity in rectangular cavities or capillaries in the center of cylindrical cavities ${ }^{20}$. (An interesting and sometimes useful variant was making in vivo measurements of the tail of a mouse, which could be measured at X-Band because it lacks lossy tissue.)

As described in the history of HMS laboratories' studies of free radicals ${ }^{573}$, we established methods to make measurements under conditions that more closely resembled those of functioning systems and did not unacceptably perturb the function and viability of the cells. Our initial studies on oxygen in cells followed the rate of consumption of oxygen, taking advantage of the ability of EPR oximetry to make measurements of oxygen in very small samples.

Using the line shape of ${ }^{15} \mathrm{~N}$ PDT to follow oxygen levels, we measured consumption of oxygen in macrophages under three conditions: at baseline, when stimulated by engulfing zymosan, and when exposed to endotoxin. We also studied the influence of endotoxin on oxygen consumption on Chinese Hamster Ovary (CHO) cells and cells from the medulla and cortex of kidneys ${ }^{201}$. We also studied the impact on oxygen consumption (a) in porcine aortic endothelial cells ${ }^{316,317}$ to understand the toxicity of arsenite and (b) in Molt-4 cells to investigate the pathophysiology of mitochondrial genetic disease ${ }^{360}$. We also carried out several studies of the effects of oxygen on the metabolism of nitroxides as described in Sect. 2.2.1.

\subsubsection{Intracellular Oxygen}

We focused extensively on measuring the biologically most important site of oxygen, i.e., the intracellular compartment, and especially on whether its oxygen concentration differed from the extracellular concentration.

I quickly became aware of two very different and contradictory points of view regarding the possibility of gradients between extracellular and intracellular oxygen, each being so obvious to its proponents that 'no one could question their conclusion'. In one line of reasoning, proponents argue that, because oxygen is so lipophilic (it partitions about 10-1 into lipid domains vs. aqueous) and membranes contain so much lipid, oxygen should be readily able to enter cells. Therefore, the supply of oxygen on both sides should quickly equilibrate such that there should be no gradient between intracellular and extracellular oxygen. This expectation was reinforced by studies by others in which it was shown that the rate of respiration of isolated mitochondria or even whole cells seemed to have a dependency on oxygen that was the same with or without the presence of the membrane [1].

In the other line of reasoning, it is equally "obvious" that, if consumption of oxygen occurs inside cells (principally in the mitochondria) and that oxygen must be 
supplied from outside the cells, then there has to be a gradient as the oxygen diffuses into the cell to replace what had been consumed/utilized.

For a while this contradiction was able to be ignored by hypothesizing a sort of compromise: while there must be a gradient, it must be very small. However, our initial paper ${ }^{71}$ suggested otherwise, i.e., that the gradient was not small. Because this finding was so revolutionary at the time, we then examined and reexamined whether we had a methodological error both by looking for potential errors in our technique and by developing alternative methods.

Our basic approach in every instance was to obtain independent measurements of both the intracellular and extracellular compartments, preferably simultaneously. In our initial paper ${ }^{71}$, we used a method based on the resolution of the hyperfine structure of a nitroxide, tempol. This nitroxide was sufficiently lipid soluble that it readily moved into the cell. We could look at the EPR spectrum of only the intracellular tempol by adding a charged paramagnetic ion to the suspension, which stayed extracellular because of the charge and broadened out the spectra of extracellular nitroxides, so as a result we saw only the intracellular signal.

To examine the extracellular oxygen content, we omitted the paramagnetic metal ion and, because the intracellular volume was very small compared to the extracellular volume with the cell suspension that was used, the spectra were essentially only those from extracellular tempol. By inhibiting oxygen consumption, we showed that the gradient disappeared.

We used a variety of approaches to verify that our findings were robust ${ }^{71,91,111}$, $149,171-173,183,195,292,360,361$. We believe that we eventually provided clear and definitive proof of the occurrence of significant gradients of oxygen and demonstrated the role of membrane organization on the extent of the gradient that occurred ${ }^{361}$. The permeability of membranes to oxygen is considered in detail by Karol Subczynski and colleagues in this special issue ${ }^{560}$.

\subsubsection{Measuring Oxygen-Dependent Phenomena In Specific Compartments/ Organs/Diseases to Study Physiology and Pathophysiology}

We recognized that the capability of EPR oximetry provided us with an exceptional opportunity to make unique and valuable contributions to the understanding of oxygen in physiology, pharmacology, and pathophsiology $y^{371,422}$. This was possible because of a combination of factors: the importance of oxygen in normal function and in disease; the unique ability of EPR oximetry using paramagnetic particulates to make repeated measurements in specific compartments and organs; and the capability of EPR to make measurements in viable, fully functioning organisms, ranging from suspensions of cells to whole animals including humans.

We, therefore, used EPR oximetry extensively to investigate the many biologically important physiological and pathophysiological processes in which oxygen has an important role. We wrote several conceptual overviews and reviews 167, 174, 203, 268, 371, 423. We carried out a series of studies that are still ongoing, most recently focused especially on direct clinical applications. (See also the paper in this special issue by Bernard Gallez [2] that summarizes other aspects of this topic.) 
The order of topics covered in the summary below is based on our published papers on the subject, as we have used our EPR techniques extensively to measure oxygen in vivo in different organs (e.g., liver) and in different compartments within tissues such as intracellular, extracellular, and intraphagosomal ${ }^{131,138,145,149,150,154,157,166,207,228,229,243,276,281,338,365-367,371 . ~}$

These studies were often based on using oximetric materials in a form that used physiological mechanisms to locate them selectively. The techniques that we used to localize included using water soluble materials which went throughout the system, using charged probes in cellular systems which remained extracellular, using small particles which localized within cells, especially phagocytic cells, and implanting large particles which stayed at the site of the injection, reflecting overall oxygen levels in the tissue.

\subsubsection{Studies of the Role of Oxygen in Phagocytosis}

Because of the importance of oxygen in phagocytosis and the importance of phagocytosis in pathophysiological processes, phagosomal oxygen was of special interest. We exploited the phagocytosing ability of the cells to incorporate the particulate

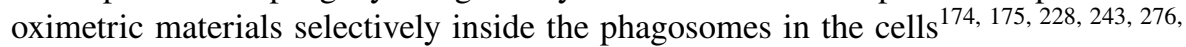

281. We especially studied the physiology and function of macrocytes, i.e., the white blood cells that are involved in responding to the presence of foreign particles such as bacteria or other particulates. This enabled us to provide unique information on the relation of the intracellular concentration of oxygen affected by the generation of superoxide from activated phagosomes ${ }^{276}$.

In the liver we were able to extend the study of oxygen and phagocytosis, to study both the process of phagocytosis in vivo and to make measurements of oxygen in a specific region of the liver. These studies took advantage of the physiologically important phagocytic capabilities of the Kupffer cells (cells in the liver that are highly phagocytic $)^{175,225,243,331,350}$. This enabled us to measure oxygen in the liver in the Kupffer cells selectively under different physiological and pathophysiological conditions and to compare their oxygen levels with other compartments in the liver, using non-specifically-locating oximetric materials.

In this series of studies, we were able to delineate the relationship of phagosomal oxygen levels in the presence of toxic events, especially those related to the presence of endotoxin (the molecule that triggers "septic shock", which includes acute hypoxia) and the oxygen dependency of the role of nitric oxide in this important clinical syndrome ${ }^{331}$. Additionally, using a different EPR oximetry approach, we were able to show that the hypoxia was induced by a lack of perfusion rather than by increased oxygen consumption, which resolved the question of the origin of the hypoxia.

\subsubsection{Studies of the Role of Oxygen in Physiology and Pathophysiology of the Liver}

We used EPR oximetry to selectively make measurements of the oxygen levels in the sinusoids of the liver and in the bulk of the liver by using two different physical forms of Gloxy: injecting a slurry, which localized in the sinusoids of the liver, 
versus injecting large particles, which remained in situ within the bulk of the liver and consequently reflected overall hepatic oxygen levels ${ }^{220,331}$. We used this approach to study the effect of modulation of endotoxin (by PR-39) to work out the mechanism of its action 220,338 .

\subsubsection{Studies of the Role of Oxygen in Physiology and Pathophysiology of the Cardiovascular System}

We carried out several studies initially in isolated hearts $184,204,207,208,211,264$, 285, 296, 373 but also in vivo (including some collaborative studies at Ohio State U.) $444,445,458,487,489,511$. Through these investigations we were able to provide unique data on the factors that result in hypoxia in the heart and on the impact of repeated hypoxic stress. The latter turned out to be potentially beneficial for decreasing the impact of a further hypoxic event.

We carried out a number of very productive preclinical studies on limb ischemia. We extended our in vivo capabilities to being able to make measurements in rabbits and studied the changes in both oxygenation and vascular flow (the latter using microspheres $)^{383}$. We found that with normal vasculature there was no direct relationship between blood flow and tissue oxygen. In contrast, with recovery from induced ischemia, there was a linear relation between blood flow and tissue oxygenation. We studied the compensating development collateral vasculature following experimentally induced limb ischemia; we looked at the return of oxygenation as collateral circulation developed, with direct measurements of oxygen in the limb providing a robust indication of the adequacy of the new vasculature ${ }^{403}$.

As we transitioned into studies for clinical applications of EPR oximetry, we recognized that perhaps the most readily exploited and clinically most effective uses of EPR oximetry will be for peripheral vascular disease (PVD) (for reasons detailed below). We were successful in obtaining funding from NIH for early feasibility studies on PVD and are currently working with several groups, especially at the U. of Maryland at Baltimore. The initial systematic clinical studies are using oximetry for diagnosis and treatment of both arterial PVD and PVD of the foot. The foot is one of the most important clinical problem areas for people with diabetes, e.g., potentially resulting in amputation.

For many of these studies we used a simple and effective method to change oxygen in tissues by having the subject breathe oxygen enriched gas mixtures. The simplicity and safety of this approach underlies much of our clinical studies, where we use the capabilities of EPR oximetry to measure these changes and thereby can improve the treatment of several major diseases including cancer and diseases related to poor circulation (ischemia) including stroke and PVD.

The preclinical studies of PVD have used charcoal ${ }^{383,402}$, LiPc, and India ink while the clinical studies have primarily used India ink $^{200,207,223,376,456,530}$. India ink has two major advantages for clinical studies. As for any use involving injection of India ink, its safe use in humans is accepted by the FDA because India ink has a history of thousands of years of use in tattoos. In addition, the location of the India ink in the superficial layers of the skin and underlying tissues is in exactly the region of clinical interest for PVD. 
The technique most likely to be adopted for clinical use in PVD will be to inject the India Ink in several sites where there is a risk of consequences of PVD, so that changes in oxygen levels can be followed over time. These changes can be used to assess the impact of therapeutic interventions to improve oxygenation and/or to follow the progression of disease. This would provide clinically crucial information that is not currently provided by any other technique.

\subsubsection{Studies of the Role of Oxygen in Cancer}

In cancer, it is widely recognized that magnetic resonance techniques have the potential to provide information on factors, especially oxygen ${ }^{328}$, that affect its diagnosis and treatment. The capabilities of NMR and EPR are in many ways complementary, as discussed above.

Our earliest studies using EPR on the oxygen effect in radiation damage were on the modification of radiation-induced free radicals by aminothiols ${ }^{5,8-10}$. While these studies did not relate directly to cancer, in a way they were the prelude to our most extensive area of study, i.e., measurements of the oxygen effect in ionizing radiation and how this might be exploited to enhance radiation therapy.

The background for the importance of measurements of oxygen in tissues in cancer patients is based on several interrelated facts ${ }^{408,409,549,569}$ :

- The response of tumors to radiation therapy is negatively impacted by the presence of hypoxic regions.

- The occurrence of hypoxia is associated with more aggressive cancers.

- Knowledge for individual patients regarding how oxygen levels in their tumors change with growth and treatment could lead to much improved treatment (e.g., by allowing clinicians to time radiotherapy to take advantage of higher levels of oxygen in the tumor with its greater response to radiation).

- EPR oximetry with particulates has some uniquely valuable characteristics for making such measurements including that measurements: directly reflect oxygen in the tumor (and other tissues), can be extended in time and repeated as needed, and (after an initial invasive step to place the oximetric materials in the tissues) can be entirely non-invasive and done quickly and comfortably.

These considerations led to an extensive set of preclinical studies in rodents $197,205,248,272,275,284,301,303,306,341,345,410$, $427,435,436,440,442,457,465,466,468,469,471,472,475,486,501-503,515,533,537$, In these studies we documented thoroughly that EPR oximetry using particulates could measure changes in oxygen in tumors and that these changes could be correlated with outcomes. Radiation delivered at times in which the oxygen was higher resulted in greater impact on the tumors (as measured by the rate of growth of the tumors and whether the animals survived). These studies also demonstrated that the use of oxygen-enriched breathing gases could significantly increase the oxygen level in tumors and lead to more impact from the irradiation. We also were able to show this with drugs that enhanced oxygenation $374,387,419,440,442,457,468$. 
Very importantly, we found that, even in tumors in inbred strains of rodents injected with tumor cells from the same source and at the same time, the oxygen levels and outcomes varied. In addition, there was significant variability of the rodents' response to interventions designed to increase oxygen in the tumors. This variability provides strong support for the need to measure oxygen in individual tumors to obtain maximum benefit.

These preclinical studies also included measurements of the effects on oxygen in tumors from other types of therapy as well as from radiation. Additionally, we studied the impacts of oxygen on key molecules involved with combined therapies such as radiation plus chemotherapy.

We believe we made the first ever measurements of the relationship between oxygen levels and responses to immunotherapy ${ }^{325}$. As immunotherapy is now being used more extensively, and evidence is accumulating that oxygen levels impact the immune response, this observation suggests that the ability to repetitively monitor oxygen provided by EPR oximetry has an immense potential for improving effectiveness of cancer therapy.

Because we have also measured the impact of other treatments on oxygen levels in tumors, this helped to clarify their mechanism of action and how they could be used optimally when combining multiple therapies. Such studies include understanding the effects when using electrochemotherapy ${ }^{351}$ and Vineblastine ${ }^{355}$. For example, since both approaches lower oxygen levels, they would interfere with the effectiveness of radiation therapy if used concurrently.

The extensive studies in preclinical models of the relationship in tumors among oxygen levels, irradiation, and impact on tumor growth led to our intensive clinical studies $207,456,490,492,496,509,516,517,530,540,547,574$. In the clinical studies, we aimed to demonstrate that the unique ability of EPR oximetry with particulates to directly make repeated measurements of oxygen within tumors could be a valuable clinical tool for patients with cancer. Our studies included direct measurements of oxygen in more than 50 human subjects. We also provided data and insights into critical aspects needed to assure the safe and effective translation of these techniques. The end goal is to alter practice to improve outcomes by providing information about oxygen levels in tumors to treating clinicians. These areas of research are continuing in collaborations with colleagues at the U. of Chicago, U. of Maryland at Baltimore, and the Catholic U. of Louvain, Brussels, Belgium.

In collaboration with Brian Pogue, we used EPR oximetry to clarify critical aspects of the mechanism of photodynamic therapy (PDT) for control of tumors $^{321,348,353,369}$. Using EPR oximetry to measure tumor oxygen levels ${ }^{321}$ and using finite element analysis ${ }^{348}$, we found evidence that the mechanism of PDT includes damage to the vasculature. We also found some evidence consistent with an impact on the consumption of oxygen ${ }^{353}$. Based on these findings we were able to show how PDT could be effectively combined with ionizing radiation ${ }^{369}$.

As summarized in the paper by Brian Pogue et al. in this special issue ${ }^{559}$, I also have begun collaborations with his group using optical methods for imaging oxygen in tumors. Brian's group has been using the phosphorescence quenching method in combination with Cherenkov-Excited Luminescence Imaging (CELI) during radiation therapy ${ }^{548,559}$. This collaboration again illustrates that there is a very valuable 
synergy when using combinations of techniques to characterize oxygen in tissues, if the basis of the measurements by each technique is understood and exploited.

\subsubsection{Studies of the Role of Oxygen in Physiology and Pathophysiology of the Kid-}

ney The kidney is another very productive area where EPR oximetry has been quite valuable. In the kidney, oxygen levels in the outer part (cortex) and the inner part (medulla) are quite different. Using particulates injected specifically into these compartments, we were able to determine where and to what extent physiological and pathophysiological processes altered oxygen concentrations ${ }^{229,271,275}$. For example, we were able to show how endotoxin altered the oxygen in the two compartments. We, therefore, could obtain insights into the pathophysiology of septic shock (which is caused by endotoxin), especially the dependency on the levels of oxygen and how nitric oxide affects the oxygen levels ${ }^{220}$. We also studied cells from the medulla and cortex in vitro and found that they also responded differently to the endotoxin ${ }^{201}$.

\subsubsection{Studies of the Role of Oxygen in Physiology and Pathophysiology} of the Brain The central nervous system, especially the brain, is another important organ system whose physiology and pathophysiology are very sensitive to levels of oxygen. EPR oximetry techniques are uniquely capable of making it possible to follow various critical aspects of brain physiology, pharmacology, and pathophysiology as these processes cause and respond to changes in oxygen levels ${ }^{375}$.

In an extensive series of studies, we were able to study the effect of anesthetics on brain oxygen $207,232,269,308,333,349,356,369,378$. Perhaps the most important result was that we showed that some of the usual injectable anesthetics (especially pentothal) used for some preclinical measurements resulted in amazingly low oxygen levels. We also showed that, with the use of inhalation anesthetics plus enriched oxygen, we could maintain brain oxygenation at normal levels. These results contributed to a general shift in the use of anesthetics in preclinical studies to inhalation anesthetics.

In another important set of studies, we used oxygen measurements in the brain to study the pathophysiology and potential treatment of cerebral ischemia (stroke) using selected obstruction of a major artery in the brain $^{326,334,335,388,407,420,463,464,499,504,532}$. These studies were especially advanced by the development of the implantable resonator (ImR). Using the ImR, we were able to obtain simultaneous, well-resolved measurements of the affected and adjacent regions as well as from the "control" side of the brain in parallel anatomical areas.

In collaboration with Manisha Patel, we determined the pattern of changes of oxygen in a critical region of the brain (hippocampus) during experimentally induced brain seizures ${ }^{428}$. Because of the ability to place the LiPc precisely in the region of interest in the brain and then to measure the oxygen levels continuously, the group was able to determine the temporal relationships of hypoxia with the products of the characteristic seizure-induced chemical changes. This helped unravel the role of these intermediates (F2-isoprostanes) in the pathophysiology of an important type of epilepsy. 
We measured the oxygenation status of the brain during adaptation to low oxygen, as occurs at high altitudes ${ }^{339}$. By combining in vivo EPR and 31P-NMR we were able to determine the relationship between fundamental aspects of oxidative metabolism and levels of oxygen, finding evidence for maintenance of function until oxygen reached a critical low level ${ }^{313}$.

We also were able to study the impact of pharmacological interventions, such as a drug that changed the affinity of hemoglobin for oxygen (Efaproxyn), which was being studied for its capability of providing oxygen to ameliorate the impact of stroke $^{334,335,374,387,419}$. Using repeated measurements of oxygen, we were able to show the relationship between dose and impact on oxygen levels and the duration of the drug-induced increase in oxygenation.

We also used a technique that we developed to measure nitric oxide in vivo (see section below) to follow the dynamics of oxygen in brain in vivo when levels of nitric oxide increased ${ }^{309}$.

\subsubsection{Studies of the Role of Oxygen in Physiology and Pathophysiology of the Skin} and Bone The skin has complex structure and functions, some of which are oxygen dependent. In particular, skin vasculature is sensitive to local oxygen levels, which in turn impact the absorption of drugs through the $\operatorname{skin}^{307,347,406}$. Another important result from our work is on the influence of different anesthetics on skin oxygenation. We presented the first direct evidence of the sensitivity of skin oxygenation to the type of anesthetic. This paralleled our pioneering studies of effects of different anesthetics on oxygenation in the brain.

In collaboration with Mark Rollins, Ralph Marcucio and other colleagues from U. of California at San Francisco, we studied the role of oxygen in the healing of fractures, providing unique insights into the mechanisms of healing of fractures ${ }^{430}$, 470,474 . We delineated aspects of the healing process that were modified by the level of oxygen and conversely how the healing process modified the levels of oxygen. The studies used LiPc injected into mice at the site of the fracture (and, as a control, in the same place of unfractured bone in the opposite leg). Data were obtained both acutely, i.e., immediately after the fracture, and then periodically over 28 days during the healing process. These studies directly demonstrated, for the first time, the details as to how oxygen levels impact the healing of fractures by influencing the vascularization. These studies, therefore, suggest that increasing the availability of oxygen could aid in the healing of fractures.

2.2.10.4 Measuring the Efficacy of Blood Transfusions The ability to provide patients with safe and effective transfusions to quickly reverse the consequences of an insufficient supply of oxygen via the circulatory system is extremely important. Currently there are many shortcomings in the available methods to do this. Most notable for our technique, there is no way to adequately evaluate the efficacy of potentially available preparations of red blood cells (RBCs) or potential substitutes for RBCs. That is, the evaluations of these preparations currently do not assess how well they deliver oxygen to tissues in vivo, which is their primary intent. In collaboration with Paul 
Buehler at U. of Maryland at Baltimore, we have begun studies to use in vivo EPR oximetry to meet this need ${ }^{535,543,545,553,565}$.

The premise is simple, but potentially could have very positive implications for enhancing the health of patients. The main reason for doing transfusions is the concern about patients having an inadequate oxygenation of tissues due to loss of oxygen-carrying capabilities of the circulatory system. By monitoring oxygen levels in subcutaneous tissue or superficial muscle, EPR oximetry could provide a simple and long term solution for determining whether RBC preparations or other approaches provide adequate oxygenation. This could be done most simply and safely by using India ink as the sensor and having a transportable EPR system. We have carried out preliminary studies in animal models that indicate that this approach is feasible. We plan now to continue these studies with studies in experimental animals to provide rigorous preclinical criteria for effectiveness of newly developed materials and by direct measurements in human subjects to demonstrate the clinical applicability.

2.2.10.5 The Role of Oxygen in "FLASH" Radiotherapy Most recently I have been involved in studies to determine if the effects of oxygen can explain a finding for an exciting new therapy called FLASH radiotherapy, in which the biological equivalent dose to conventional radiotherapy is delivered at very high dose rates. The surprising finding, known as 'the FLASH effect', is that FLASH compared to conventional radiotherapy appears to be equally effective against tumor tissue while being relative better at sparing normal tissues 562,575 . The most frequently postulated mechanism for the FLASH effect revolves around differential depletion of oxygen by FLASH versus radiation delivered at conventional dose rates, and that this depletion of oxygen protects normal tissues while maintaining effectiveness against the tumor.

To test such a hypothesis, measurements of oxygen need to capture the characteristics of the relevant crucial reactions involving oxygen. In turn, these characteristics determine the requirements for the site and speed of the measurements of oxygen. The critical site for cell death from radiation is in the nucleus, and so the relevant measurements of oxygen depletion need to be made in the nucleus. The speed is determined by the short lifetime of the radiation-induced intermediate because the effect of oxygen on radiation damage occurs by reacting with the short-lived reactive intermediate ${ }^{575}$. However, as noted in our series of papers as to what is the meaning of a measurement of oxygen ${ }^{540,541,546,549}$, while there is a need to make very fast measurements selectively in the nucleus, currently no technique can do that in vivo. We are working on developing such techniques to measure oxygen selectively in the nucleus in vivo.

2.2.10.6 Other Oximetry Studies There are a number of other publications in which we measured oxygen that do not readily fit into the categories discussed above. As a very important part of understanding the function and potential toxicity of melanins and their interactions with cells we characterized interactions of oxygen with the naturally occurring free radical in melanin ${ }^{58,159}$. (See Sarna et al. ${ }^{570}$ for a much more extensive consideration of the interactions of oxygen with 
melanins.) We also applied the technique to photosynthesis where we could follow the generation of oxygen as a measure of the metabolism of the plant ${ }^{129,164}$.

We (principally Graham Timmins) used EPR oximetry to explore the hypothesis that bioluminescence, which requires oxygen for the reactions that result in bioluminescence, may have evolved as a means to reduce tissue oxygen levels a safer value $322,329,343,357$. By carefully measuring the consumption of oxygen in fireflies and bioluminescent larvae, we found that the amount of oxygen that was consumed was too small to have significantly lowered oxygen levels. Nevertheless, in the process we had some interesting experiences as we procured and worked with these exotic samples! We also discovered that fireflies have a mechanism to control the availability of oxygen, which is interesting in itself but also has implications that support the existence of barriers to oxygen in mammalian cells.

In collaboration with a group from Tbilisi, Georgia, we used in vivo EPR oximetry in mice to help characterize the mechanism of a quinoid "radio-toxin", which the group felt had effects analogous to those of ionizing radiation ${ }^{426}$. We measured the impact of various doses on the time course of oxygen in the liver and the brain of mice, finding that potentially lethal doses of the toxin resulted in a dysregulation of the supply of oxygen, leading in turn to hypoxia.

In collaboration with Aria Tzika from Harvard Medical School (also sometimes abbreviated as HMS!), we used EPR oximetry and EPR methods to study redox related parameters to investigate the mechanism of the pathophysiology of trauma and burns $434,485,512$. These measurements were correlated with those made with NMR. These experiments led to a conclusion that mitochondria-targeted antioxidants promote recovery of skeletal muscle mitochondrial function after trauma, which has significance for the treatment of severe trauma.

In collaborations we also carried out some studies on oxygen that did not use $\mathrm{EPR}^{342,562}$. We especially were interested in modeling the distribution of oxygen and carried out a study using the Eppendorf polarograph and modeling with numerical simulations to explain the results ${ }^{342}$.

2.2.10.7 Measurements of Nitric Oxide and Oxygen Nitric oxide is a potent modulator of oxygen in vivo, both because of its direct reactions with oxygen-centered radicals and as a modulator of the function of the vascular system (It causes blood vessels to dilate, thereby increasing blood flow which in turn increases the availability of oxygen in tissues).

In order to measure nitric oxide in vivo, we adapted the known interactions of the chelator diethyldithiocarbamate (DETC) and nitric oxide, which has a characteristic EPR signal, parts of which do not overlap with oxygen sensors such as Gloxy $^{290,309,350,364}$. We therefore were able to measure both oxygen and nitric oxide simultaneously in vivo and study the effect of nitric oxide on tissue oxygen in brain and liver.

2.2.10.8 Measurements of Redox Status There are many very important physiological and pathophysiological processes that are impacted by redox status of 
tissues, but there is a paucity of techniques available to do this, especially in vivo. EPR provides some unique information for studying redox status ${ }^{363,367}$. Measurements of redox status are closely linked to measurements of oxygen in tissue. Because they are mechanistically linked, measurements of one usually provide important information about the status of the other. We found that in many circumstances it is optimal to use EPR to measure several different but complementary parameters, such as the measurement of available sulfhydryl groups, reactive nitrogen species, redox dependent metabolism (e.g., level of metabolic free radical intermediates, rate of reduction of nitroxides, and rate of oxidation of hydroxylamines), and oxygen in tissue $363,431,434,485,512$.

Our studies measuring SH groups in living systems involved collaborations with two outstanding researchers from other institutions, Lev Weiner and Valery Khramstov $^{168,379,431}$. Our development of the use of nitroxides for measuring redox status are reviewed elsewhere in this paper and in an accompanying $\operatorname{paper}^{573}$.

\subsection{Methods to Measure Oxygen}

\subsubsection{Instrumental/Technique Developments to Facilitate Epr Oximetry, Including for Clinical Use}

The data needed to reach the biological goals of my laboratories' studies was a strong driving force for the development of instrumentation that would enable EPR measurements to be made in functioning biological systems under well-controlled conditions. (Developments as of 2003 were summarized in a book in the Biological Magnetic Resonance series ${ }^{318}$.) In turn as these technical capabilities were developed, they enabled additional types of studies to be carried out.

This is most dramatically illustrated by the initial development of in vivo EPR to advance oximetry ${ }^{319}$, which then led to the in vivo biodosimetry capabilities that have been a large focus of our laboratory ${ }^{571}$. Similarly, the instrumental developments for studying free radicals in functioning biological systems ${ }^{573}$ led to the capabilities that helped advance oximetry. The instrumental and technical developments for oximetry in cellular systems and in vivo were quite different. Although we quickly moved to lower frequency EPR at L-Band $(\sim 1 \mathrm{GHz})$ which enabled us to make measurements within the body, we started out in vivo EPR at X-Band $(\sim 9 \mathrm{GHz})$ using the tail of the mouse inserted into the X-Band resonator ${ }^{139}$ and using a paramagnetic NMR contrast agent.

\subsubsection{Development of Oxygen Sensitive Materials and Systems}

Our studies that centered on methods to measure oxygen began with an article entitled "Measurement of intracellular oxygen concentration using the spin label TEMPOL". As has been the case with most techniques to measure oxygen, our development of EPR oximetry was closely coupled with the biological question that was 
being pursued, rather than on engineering related improvements of the measurements per se.

The methodology that was employed was based on the impact of the paramagnetic oxygen molecule on EPR spectra of all paramagnetic materials, as laid out by others in previous publications [3-5]. There are several different experimental approaches to utilize this phenomenon, including impact on the overall line width, impact on the resolution of hyperfine structure, and impact on relaxation times ${ }^{58,366}$.

The common basis is the use of a fundamental physical principle, the Heisenberg Uncertainty Principle, that says that there is a fundamental limit on the resolution of paired phenomena such as lifetime and position. So, in the case of EPR lines, the more precise you know the lifetime of the state, the less you can know about its position, which manifests itself as a broadening of the EPR spectral line. Although in principle all unpaired electron species could be used for oximetry, there are several approaches that we and others have used to enhance the effectiveness of these measurements, which we summarized in several publication ${ }^{104,366,369}$. In general, having a very narrow line width is an important principle so that small changes in oxygen can be detected.

We systematically attempted to develop paramagnetic materials that would be especially useful for oximetry ${ }^{163,365,366}$. We built on early studies to optimize nitroxides, including the use of isotopic substitutions to narrow the line width. For example, in nitroxides, hydrogen atoms were replaced with deuterons, because of the smaller magnetic moment of the deuterons, and/or were replaced with $\mathrm{N}^{15}$. In collaboration with Steve Bottle, we carried out a series of studies to optimize the structures of nitroxides to combine stability in vivo with sensitivity to oxygen ${ }^{355,467}$.

While the nitroxides provide a powerful tool to measure oxygen and oxygendependent metabolism, it is unlikely that they will be used in human subjects in the foreseeable future, because they would require the very time-consuming and expensive process to obtain premarketing approval of a new drug by the FDA.

We made several studies in animals using India ink, especially because of its potential to be used in human subjects without a need to go through the long and expensive FDA process ${ }^{175,207,200,223,225}$. Our studies with India ink built upon the extensive studies of India ink pioneered by Bernard Gallez [2]. Based on the successful experience in animals we then carried out pilot studies in human subjects who had preexisting India ink in their skin from tattoos. We then optimized India inks prepared to be suitable for oximetry in human subjects (after testing to assure their sterility and lack of toxic components). We then used these inks to carry out several studies in human subjects, with a focus on PVD and cancer $207,456,490,496,530$.

We worked with the French group that had developed lithium phthalocyanine (LiPc) as a narrow line width paramagnetic material for other uses [6]. (They had developed it as part of a magnetometer designed to detect submarines!) We characterized its oximetric properties, which turned out to be very excellent for use in viable biological systems for three reasons: LiPc has high sensitivity due to it high stability in tissues, a narrow line width, and a high number of spins ${ }^{170,176,}$ $188,312,404$. This eventually led to clinical trials in which we carried out the first-inhuman systematic study of clinical oximetry based on a PDMS-coated derivative of $\operatorname{LiPc}^{551,560,574}$ 
In collaboration with Bernard Gallez and Bob Clarkson, we also developed carbon-based materials other than India inks (coals, charcoals, and chars). We studied a wide range of coals and charcoals, investigating the basis of their response to oxygen ${ }^{418}$. We eventually found that a coal termed "fusinite" was especially

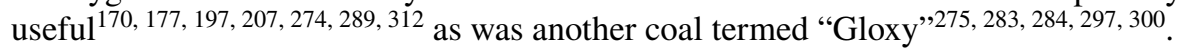

We also carried out systematic studies on the effects of the oximetric materials on the tissues and in general found them to have very low toxicity $^{70,176,194,207,270,283,312,324 .}$

In collaboration with Bernard Gallez, we pioneered the use of oxygen permeable biocompatible coverings to facilitate the use of our particulates in human subjects and have continued these developments at Dartmouth ${ }^{274,289,340,346,404,439,518}$. While the particulates do have generally low toxicity, the use of encapsulation in biocompatible materials that are already cleared for use in human subjects was a very important step that enabled much earlier clinical use of EPR oximetry with materials in addition to India ink. The requirement for use of the oxygen-sensitive materials was thereby transformed from the need to show that the materials were safe and effective in humans (a process that can require many years and more than $\$ 500$ million) to only needing to show that the material remained fully encapsulated. We also developed an alternative encapsulating material, albumin microspheres, which had the property of retaining nitroxides, i.e., protecting them from metabolism, while retaining their full ability to measure oxygen ${ }^{220}$.

\subsubsection{Comparisons of EPR Oximetry with Other Techniques}

It is very important to demonstrate that the oxygen measurements with EPR are consistent with those made with other validated methods. We were able to show that EPR oximetry compared favorably in direct comparisons with oxygen electrodes 245 , ${ }^{380}$, NIR measurements ${ }^{377}$, and fluorescent quenching with the OxyLite apparatus ${ }^{393}$. More generally we wrote several articles in which we summarized and compared various techniques to measure oxygen in living systems, especially in relation to $\mathrm{EPR}^{220}, 280,294,295,336,421$. We especially carried out parallel NMR and EPR studies of oxygen dependent phenomena, adding to the utility of both techniques. (These studies are summarized in a prior section.) While most other techniques do not provide direct measurements of oxygen in the tissues, they often do provide different complementary information that, together with EPR oximetry, may lead to better overall understanding of the pathophysiology.

\subsubsection{Development of in vivo EPR Oximetry Spectrometers}

We have spent a major amount of effort on the development of in vivo EPR spectrometers for oximetry especially, but also for other applications as well. This emphasis was rooted philosophically in the concept that, while model systems and cellular systems can be quite productive and often easier to control, in order to fully understand biological phenomena it is essential to make rigorous studies in vivo. Only with in vivo measurements can one be confident that all of the factors that can 
moderate physiological and pathophysiological phenomena are potentially active. It was also rooted in my long-term goal of clinical applications of EPR.

Consequently, we have produced many papers on the development of in vivo EPR and especially on clinical applications of EPR. As it turns out, the most important clinical application is oximetry. That is because of the very major role that oxygen has in physiology and pathophysiology as well as the almost unique ability of EPR to make the measurements directly in the tissues and to make them repeatedly and non-invasively (after initial placement of the EPR materials in tis-

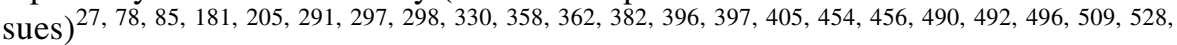
537,540

The development of optimized resonators was one of the keys for effective in vivo EPR, as reviewed in detail in a paper in this special issue ${ }^{555}$. Two approaches have been especially important, surface resonators and implantable resonators (ImR). For small animals such as mice we also developed whole body resonators that enveloped the animals ${ }^{224}$. The surface resonators have the advantage of being applicable to any sized object, but they have the limitation of being restricted to measurements within about $10 \mathrm{~mm}$ of the sample's surface. Nevertheless, these have been very useful in some clinical measurements of oxygen, especially for PVD and tumors near the surface such as melanomas.

The current capabilities of the surface resonators have been summarized in two papers in this special issue ${ }^{555,561}$. The ImRs have been developed over a long period of time and have been very effective for preclinical applications. Recently these have been further developed with a focus on clinical applications ${ }^{531,} 568$. With the new designs and methods for fabrication, it seems very probable that the ImR will be the prime approach for clinical oximetry for all tumors except those located quite superficially. This is an area in which I am intensively participating and expect to continue to do so for the foreseeable future.

While I always had a long-term aim to have my research impact the effectiveness of clinical medicine, the development of EPR instruments at low frequency provided an especially attractive possibility of making direct measurements in human subjects to obtain information that would impact the health of the individual.

However, making EPR instruments suitable for clinical applications raises several technical and conceptual challenges, as we articulated elsewhere ${ }^{268}$. Briefly, the instrument needs to be suitable to accommodate sick human subjects, which requires both its size and procedures to allow measurements to be made quickly, safely, and comfortably. Equally important, we needed to be able to provide information that the clinicians would feel was valuable and present it to them in a form that they could readily use. We also needed to make the instruments suitable for use in a clinical setting where the measurements would be made and operated by personnel already associated with the clinical setting. Its operation needed to be accomplished by them sufficiently quickly and readily so as to not impede clinical throughput or efficiency.

While there are many further refinements that are needed and some are in process, I think that we have achieved our goal of making clinical EPR fully feasible. Attaining this goal for direct medical applications of EPR (principally measuring 
oxygen) was greatly facilitated by our experience in making EPR instruments for dosimetry ${ }^{571}$. The dosimetry developments were important both in terms of the instruments that needed to be developed and in providing a strong financial basis for employing and maintaining a creative group of engineers and EPR spectroscopists.

\section{Conclusions on the Future of EPR Oximetry}

\subsection{The Unique Roles of in EPR Oximetry in Biology and Medicine}

There is a very bright future for this technique, which derives from some special (and in some respects unique) capabilities of EPR oximetry. For the type of EPR oximetry that we have pursued (based on particulates), the exceptional capabilities include that the measurements can be made directly in the tissues, can be repeated as often as desired over short and long time periods, and are non-perturbing. EPR imaging and simultaneous measurements of multiple EPR spectra adds the potential for resolving the spatial distribution of oxygen.

These capabilities are essentially unique in existing clinical methods to measure oxygen in tissue. In fact, oxygen levels in the tissues of interest are not measured directly with many of the most commonly used methods such as the NMR-based techniques (including BOLD, measurements of perfusion, diffusion weighted measurements), PET-based measurements of hypoxia sensitive molecules such as misonidazole, Doppler measurements of perfusion, and optical measurements of hemoglobin saturation. Instead, these methods measure parameters related to oxygen in tissues but by themselves cannot provide well-founded information on oxygen levels in tissues or how they change. While there are a few methods that also can measure oxygen directly in tissue including NMR oxygen sensitive reagents injected directly in tissues, the oxygen electrode, and some optical methods, none of these can be repeated and none of them has entered systematic clinical research use, in contrast to EPR oximetry with particles.

\subsubsection{Animal Models}

For use in animal models, it seems clear that EPR oximetry has become an extremely valuable and well-recognized method to obtain very useful data on oxygen that cannot be obtained by other methods. In addition, in animals we also can use the full range of techniques to study redox phenomena employing nitroxides and spin traps. This very significantly expands the scope and importance of the studies directly and indirectly related to oxygen pathophysiology. 


\subsubsection{Human Subjects}

As noted above, studies in human subjects require meeting regulatory challenges and also ergonomic issues to be able to be used safely and effectively in patients.

India ink (i.e., suspended carbon-based particulates) is an important exception to the full regulatory challenges because it has been used so long in humans (as tattoos for cosmetic purposes and in medical marking) that the FDA has ruled that it is exempt from needing further approval for its injection in humans ${ }^{570}$. For other oximetric materials, it is likely that they will need to be used with biocompatible coatings, but this should not be limiting in the long run ${ }^{537}$.

Another and perhaps greater challenge is the depth at which in vivo oximetry measurements can be made. For India ink, the maximum depth is only a few mm. This is sufficient for some uses such as for superficial tumors like melanomas and, most importantly, for PVD where EPR oximetry may have a very important role in the future. However, for most tumors, the EPR approach of choice will be using the implantable resonator which provides excellent signal/noise at any depth and is designed to obtain data from several sites simultaneously. It should be very feasible to move the current research oriented EPR oximeter that has already made many valuable measurements of oxygen in patients into an instrument that can be widely utilized on a routine basis for optimizing treatment in oncology and PVD. This is the direction that I am currently pursuing.

\subsection{The Role of EPR to Find Clinically Meaningful Measures of Oxygen for Their Intended Use}

Finally, and perhaps most importantly, we have begun to focus on what is the meaning of a "measurement" of oxygen. Previously we had carried out systematic comparisons of what is measured by various types of "measurements of oxygen"336, 372, 421. Our new overall conclusion, summarized in a recent series of papers, is that ALL types of clinical measurements of oxygen do not really provide an 'absolute value' of oxygen, because no method can resolve the heterogeneity of oxygen in both normal and pathological conditions $540,541,546,549,569$. These considerations have a very large impact on the clinical use of oximetry including on how to carry out the measurements and how to interpret the results that have been obtained. This leads to several important and useful conclusions:

- The "absolute" measurement of oxygen levels in tissues is almost always actually a value that averages a range of absolute values of oxygen in the tissue.

- If you want to measure oxygen levels in tissues, you need to measure directly in those tissues.

- All currently clinically available techniques for measurements of oxygen in tissues do not directly measure oxygen in the tissues and therefore need to be understood as providing indirect measures.

- EPR oximetry with paramagnetic particulates injected into tissues can provide such measurements. 
- EPR oximetry can be used to determine the circumstances where indirect methods accurately reflect oxygen levels in the tissues.

- For most clinical applications, repeated measurements of changes of oxygen levels in tissues are likely to provide information that is very useful for decision making for clinical care.

EPR oximetry indeed has already established that it is directly assessing oxygen in tissues and can be repeated indefinitely. On this basis, the most important conclusion is that EPR oximetry will have a very bright future as a clinically important technique in humans. It also will continue to be very valuable for studies using animal models and for some cell systems.

Acknowledgements These studies and instrumental developments are the product of many people's efforts as well as funding from many sources over the past fifty or so years. While I have named several special contributors in the context of this history, I probably have not been thorough, so the reader should note the many coauthors of my papers for a fuller picture of the key players.

Editors' Note Because there is considerable overlap in the references in the three histories of EPR studies from H.M. Swartz laboratories, references with Swartz as an author use a common numbering system. These numbers are from HMS' curriculum vita and are noted by superscript numbers in this text. The full references are published only in the accompanying editorial, Issue 2 of the Special Issues of AMR on the Occasion of Harold M. Swartz's 85th Birthday [8]. References included below with this history are those that do not include Swartz as a coauthor.

\section{Declarations}

Conflict of interest Harold Swartz and Ann Flood are co-founders of Clin-EPR, LLC which makes in vivo EPR instruments for preclinical and clinical investigational use only.

\section{References}

1. B. Smaller, E.C. Avery, Nature 183, 539 (1959)

2. D.P. Jones, H.S. Mason, J Biol Chem 253, 4874 (1978)

3. B. Gallez, Appl Magn Reson 52, 1395 (2021). https://doi.org/10.1007/s00723-021-01358-7

4. M.J. Povich, Anal Chem 47, 346 (1975)

5. C.A. Popp, J.S. Hyde, J Magn Reson 43, 249 (1981)

6. J.N. Backer, V.G. Budker, S.I. Eremenko, Y.N. Molin, Biochim Biophys Acta 460, 152-156 (1977)

7. P. Turek, J.-J. Andre, A. Giraudeau, J Simon Chem Phys Lett 134, 471-476 (1987)

8. A.B. Flood, S.G. Swarts, M.C. Krishna, B. Gallez, Appl. Mag. Res. In Press (2022). https://doi.org/ 10.1007/s00723-021-01459-3

Publisher's Note Springer Nature remains neutral with regard to jurisdictional claims in published maps and institutional affiliations. 